\title{
Gene regulation of anthocyanin biosynthesis in two blood-flesh peach (Prunus persica (L.) Batsch) cultivars during fruit development ${ }^{* \#}$
}

\author{
Yun JIAO ${ }^{\dagger}$, Rui-juan MA, Zhi-jun SHEN, Juan YAN, Ming-liang YU ${ }^{\dagger \neq}$ \\ (Jiangsu Key Laboratory for Horticultural Crop Genetic Improvement, Institute of Horticulture, \\ Jiangsu Academy of Agricultural Sciences, Nanjing 210014, China) \\ †E-mail: jydyx@163.com; mly1008@aliyun.com \\ Received Mar. 21, 2014; Revision accepted July 20, 2014; Crosschecked Aug. 22, 2014
}

\begin{abstract}
The blood-flesh peach has become popular in China due to its attractive anthocyanin-induced pigmentation and antioxidant properties. In this study, we investigated the molecular mechanisms underlying anthocyanin accumulation by examining the expression of nine genes of the anthocyanin biosynthesis pathway found in the peach mesocarp. Expression was measured at six developmental stages in fruit of two blood-flesh and one white-flesh peach cultivars, using quantitative reverse transcription polymerase chain reaction (qRT-PCR). Results show that the expression of the chalcone synthase $(\mathrm{CHS})$ gene was closely related to anthocyanin accumulation in both of the blood-flesh peaches. In the white-flesh peach, we found that the transcription level of phenylalanine ammonia-lyase $(P A L)$ during fruit development was much lower than that in the blood-flesh peach, even though all other genes of the anthocyanin biosynthesis pathway were highly expressed, suggesting that the PAL gene may be limiting in anthocyanin production in the white-flesh peach. Moreover, the transcription levels of the CHS and UDP-glucose-flavonoid 3-O-glucosyltransferase (UFGT) genes were markedly up-regulated at three days after bag removal (DABR) in the blood-flesh peach, suggesting that CHS and UFGT are the key genes in the process of anthocyanin biosynthesis for both of the blood-flesh peaches. The present study will be of great help in improving understanding of the molecular mechanisms involved in anthocyanin accumulation in blood-flesh peaches.
\end{abstract}

Key words: Blood-flesh peach, Anthocyanins, Bagging, Gene expression doi:10.1631/jzus.B1400086

Document code: A

CLC number: S662.1

\section{Introduction}

Anthocyanins synthesized by the phenylpropanoid pathway have been well characterized in different plant species (Pandey et al., 2014). The major anthocyanins include pelargonidin (orange or red), cyanidin (pink or red), and delphinidin (purple or blue)

\footnotetext{
* Corresponding author

" Project supported by the China Agriculture Research System (No. CAR-31), and the Jiangsu Agriculture Science and Technology Innovation Fund (No. CX(15)2014) of China

\# Electronic supplementary materials: The online version of this article (http://dx.doi.org/10.1631/jzus.B1400086) contains supplementary materials, which are available to authorized users

(c) Zhejiang University and Springer-Verlag Berlin Heidelberg 2014
}

and their formation depends on their hydroxylation pattern (Niu et al., 2010). Anthocyanins, as well as other health-promoting phytonutrients, are present in relatively large amounts in fresh fruit, and their bright colors help to make them more attractive to the consumer. Moreover, anthocyanins have been found to have strong antioxidant properties and are thought to reduce the incidence of certain cancers and heart disease (Dragsted et al., 2006; Shin et al., 2006; Butelli et al., 2008; Williams et al., 2008). Thus, the investigation of anthocyanin biosynthesis in fruit has become a very popular area of research.

The blood-flesh peach [Prunus persica (L.) Batsch] belongs to the Rosaceae family. Some peach 
cultivars exhibit fruits with red pigmentation of the mesocarp around the stone that generally appears during the later stages of fruit ripening (Hsia et al., 1965; van Blaricom and Senn, 1967; Shen et al., 2013; Zhou et al., 2013). The Canadian peach cultivar 'Harrow Blood' was the first to exhibit a fully blood-flesh peach, producing fruits with purple pigmentation of the mesocarp and skin, associated with a red midrib color on the abaxial side of the leaf (Chaparro et al., 1995). Anthocyanin accumulation was found to be initiated early, at about $50 \mathrm{~d}$ after full bloom (DAFB) (Shen et al., 2013). A similar profile for anthocyanin accumulation was observed in the skin and flesh of 'Stark Red Gold' nectarines (Ravaglia et al., 2013). Taken together, these cultivars provide interesting models for dissecting the mechanisms involved in anthocyanin accumulation.

Plant anthocyanin biosynthetic genes have been identified or cloned in many plant species, e.g., apples (Takos et al., 2006; Chagné et al., 2007; Espley et al., 2007), pear (Zhang et al., 2011; Yu et al., 2012), and Chinese bayberry (Niu et al., 2010). Of the genes discovered, phenylalanine ammonia-lyase $(P A L)$, chalcone synthase $(\mathrm{CHS})$, chalcone isomerase $(\mathrm{CHI})$, flavanone 3-hydroxylase $(F 3 H)$, dihydroflavonol 4-reductase $(D F R)$, anthocyanin synthase (ANS), and UDP-glucose-flavonoid 3-O-glucosyltransferase (UFGT) have all been found to participate in the biosynthesis of anthocyanin. In addition, transcription factors (TFs) that regulate anthocyanin biosynthesis have been identified and characterized in several plant species (Allan et al., 2008). For instance, the TFs of anthocyanin biosynthesis in Arabidopsis were found to contain an R2R3-MYB domain, a basic helix-loop-helix (bHLH) domain, and WD40 repeats (WDRs) (Morita et al., 2006) that work together to control the expression of the gene involved in the catalysis of the biosynthesis of anthocyanin (Baudry et al., 2004; 2006; Lepiniec et al., 2006). Similarly, the induction of anthocyanin biosynthesis in transient assays using MYB10 is dependent on the co-expression of the bHLH3 domain in peach (Ravaglia et al., 2013) and apple (Espley et al., 2007).

In this paper, we report on a study of the molecular mechanisms of anthocyanin synthesis examining seven enzyme-encoding genes and two TFs involved in the anthocyanin biosynthetic pathway of two blood-flesh peach cultivars during fruit development.

\section{Materials and methods}

\subsection{Plant materials}

We collected fruit of two blood-flesh peach cultivars, 'Beijingyixianhong' and 'Heiyoutao', and one white-fleshed peach, 'Xuebaitao'. All peaches were collected during the growing season (from March to August, 2013) from an orchard located in the germplasm resource center of peach (Nanjing, China). The sampling times of 'Beijingyixianhong' were 50 (S1), 64 (S2), 72 (S3), 80 (S4), 82 (S5), and 87 (S6) DAFB, while the sampling times of both 'Heiyoutao' and 'Xuebaitao' were 60 (S1), 74 (S2), 88 (S3), 97 (S4), 109 (S5), and 123 (S6) DAFB. For fruit bagging experiments, similarly-sized trees were randomly divided into three groups (replicates), with three trees in each group. The fruit in each group was covered with double layer (yellow outside and black inside) paper bags (Yunong (Qingdao) Co., Ltd., China) at 55 DAFB. Bags were removed at 72 DAFB for 'Beijingyixianhong' and 88 DAFB for 'Heiyoutao' and 'Xuebaitao', and the fruit was harvested at 0, 3, 5, 10, and $15 \mathrm{~d}$ after bag removal (DABR). Fruit skin (about $1 \mathrm{~mm}$ of the cortical tissue) and flesh samples (divided into two parts) were collected separately from at least 12 replicate fruit. In a separate experiment, fruit skin (FS), young leaves (YL), petals (P), and young stems (YS) were sampled at 60 DAFB to test the tissue-specific expression of the genes. For all sampling times, tissues were immediately frozen in liquid nitrogen and stored at $-80{ }^{\circ} \mathrm{C}$ for further use.

\subsection{Extraction and measurement of anthocyanin}

Frozen fruit $(3 \mathrm{~g})$ was homogenized with $7.0 \mathrm{ml}$ of a solvent containing methyl alcohol/water/formic $\mathrm{acid} /$ trifluoroacetic acid $(70: 27: 2: 1, \mathrm{v} / \mathrm{v})$ for $24 \mathrm{~h}$ in the dark. The separations were centrifuged $(12000 \mathrm{r} / \mathrm{min}$, $\left.20 \mathrm{~min}, 4^{\circ} \mathrm{C}\right)$, filtered $(0.22 \mu \mathrm{m})$, and analyzed via an Agilent 1100 series high performance liquid chromatography (HPLC) system (Agilent, USA). Samples $(10.0 \mu \mathrm{l}$ of extract) were further analyzed using a ZORBAX SB-C ${ }_{18}$ column (4.6 mm $\left.\times 250 \mathrm{~mm}, 5 \mu \mathrm{m}\right)$ coupled with an ultraviolet (UV) detector at $515 \mathrm{~nm}$ with a flow rate of $0.8 \mathrm{ml} / \mathrm{min}$ at $35{ }^{\circ} \mathrm{C}$. The mobile phase was a linear gradient of $10 \%$ aqueous formic acid (solvent $\mathrm{A}$ ) and formic acid/acetonitrile (85:15, v/v; solvent B). Conditions: initial 95\% A, 5\% B; in $30 \mathrm{~min}$ to $88 \% \mathrm{~A}, 12 \% \mathrm{~B}$; in $40 \mathrm{~min}$ to $75 \% \mathrm{~A}, 25 \% \mathrm{~B}$; 
in 50 min to $70 \% \mathrm{~A}, 30 \% \mathrm{~B}$; in $60 \min$ to $95 \% \mathrm{~A}, 5 \%$ B. Finally, the compounds were quantified by comparing the peak areas and presented as mg of cyanidin3-glucoside and cyanidin-3-rutinoside (Sigma-Aldrich, USA) per $100 \mathrm{~g}$ fresh tissue.

\subsection{RNA extraction and quantitative reverse tran- scription polymerase chain reaction (qRT-PCR)}

Total RNA was extracted from each sample using the EASY spin plant RNA extract kit (Biomed, Beijing, China). RNA was digested with DNase I and subsequently reverse-transcribed using a Supermo III RT kit (BioTeKe, Beijing, China). Samples of cDNA were diluted to $1: 10$ with $\mathrm{dH}_{2} \mathrm{O}$ for use in qRT-PCR. All gene-specific primers were designed using Primer3 (http://frodo.wi.mit.edu/cgibin/primer3/primer3_www. cgi), according to the available databases (http://www ncbi.nlm.nih.gov/Genbank/index.html) and published papers (Espley et al., 2007; Lin-Wang et al., 2010; Zhou et al., 2013) (Table 1). The specificity of each primer pair was analyzed by a melting curve at the end of the PCR run, with one single peak and no dimer formation. The specificity of these primers was confirmed by cloning and sequencing of qRT-PCR products. We conducted qRT-PCR using an ABI 7500 System (Applied Biosystems, Foster, CA, USA) with the SYBR Premix Ex Taq ${ }^{\mathrm{TM}}$ (TaKaRa, Dalian, China). The qRT-PCR reaction solution consisted of $10 \mu \mathrm{l}$ of SYBR Green PCR Master Mix, $0.4 \mu$ forward primer $(10 \mu \mathrm{mol} / \mathrm{L}), 0.4 \mu$ reverse primer $(10 \mu \mathrm{mol} / \mathrm{L})$, $0.4 \mu \mathrm{l}$ ROX Reference dye (50×; all from TaKaRa),

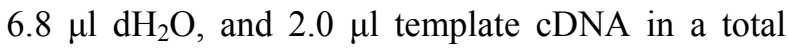
volume of $20 \mu \mathrm{l}$. The qRT-PCR amplification procedure was initiated with a preliminary step of $95{ }^{\circ} \mathrm{C}$ for $30 \mathrm{~s}$, followed by 40 cycles of $95{ }^{\circ} \mathrm{C}$ for $5 \mathrm{~s}$ and $60{ }^{\circ} \mathrm{C}$ for $34 \mathrm{~s}$. All qRT-PCR reactions were normalized using the housekeeping genes $U B Q 10$ and TEF2 (Tong et al., 2009). Relative gene expression levels were calculated using the $2^{-\Delta \Delta C_{\mathrm{T}}}$ method (Livak and Schmittgen, 2001) with three measurements for each biological replicate sample.

\subsection{Statistical analysis}

All statistical analyses were performed using the Data Processing System (DPS, Version 14.10; Zhejiang University, Hangzhou, China). All results were summarized as mean \pm standard deviation (SD); least significant differences (LSDs; $\alpha=0.05$ ) were considered statistically significant. The graphs were drawn with Origin software version 8.1 (Microcal Software Inc., Northampton, MA, USA).

\section{Results}

\subsection{Anthocyanin content in blood-flesh peach during fruit development}

The total anthocyanin content, comprising cyanidin-3-glucoside and cyanidin-3-rutinoside, varied greatly among different tissues of 'Heiyoutao' and 'Beijingyixianhong' during fruit development (Figs. 1 and 2). The anthocyanin content of 'Heiyoutao' increased significantly from 60 to 88 DAFB and declined thereafter. Anthocyanin was not detected until 64 DAFB in 'Beijingyixianhong' and then increased continuously from 64 to 87 DAFB before reaching its maximum. The highest content of anthocyanin was found in the fruit skin, followed by the outer layer of

Table 1 Primers used for RT-PCR analysis

\begin{tabular}{llllc}
\hline \multicolumn{1}{c}{ Gene } & GenBank ID & \multicolumn{1}{c}{ Forward primer (5' to 3') } & \multicolumn{1}{c}{ Reverse primer (5' to 3') } & $\begin{array}{c}\text { Product } \\
\text { size (bp) }\end{array}$ \\
\hline$P p P A L$ & HM543574.1 & AAGCTGCTGAAAAGGTGCAT & TCATTTTGGTTGCTGCTCTG & 126 \\
$P p C H S$ & HM543568.1 & CCGTGAAGTTGGGCTTACAT & TGGGTGTGCAATCCAGAATA & 139 \\
$P p C H I$ & HM543569.1 & AATATGGGGTGCAGCTTGAG & TGCTGGAAAATGAAACGTGA & 155 \\
$P p F 3 H$ & HM543570.1 & CAAAACCCAGCTCAAGAAGC & CTCCGAGTCCTGCAATTGT & 174 \\
$P p D F R$ & HM543571.1 & TGAATGTCGAAGAGCACCAG & AAATGGGCCAATCACAAGAG & 197 \\
$P p U F G T$ & JX149550.1 & TGGCCTCTCAAGGACAGTT & CCGCCTGCTATAGTCTCCAG & 173 \\
$P p A N S$ & HM543572.1 & AGGAGTTGAAGAAGGCAGCA & GCTCAATGGGAAGATCGAAA & 123 \\
$P p M Y B 10$ & EU155160.1 & GGCAGGACTGCGAATAATGT & CCCACCAATCACGTTGAGTA & 196 \\
$P p T T 8$ & KF585000.1 & CCGACCACCCACATTTCC & TGACCGTTGCGGCCCGTCTC & 163 \\
$T E F 2$ & TC3544 & GGTGTGACGATGAAGAGTGATG & TGAAGGAGAGGGAAGGTGAAAG & 129 \\
$U B Q 10$ & TC2782 & AAGGCTAAGATCCAAGACAAAGAG & CCACGAAGACGAAGCACTAAG & 146 \\
\hline
\end{tabular}


(a)
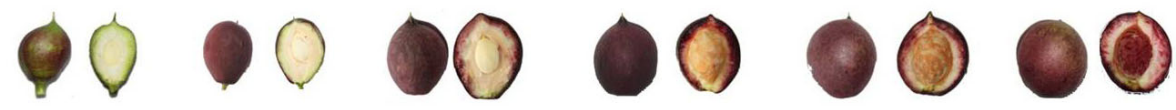

(b)
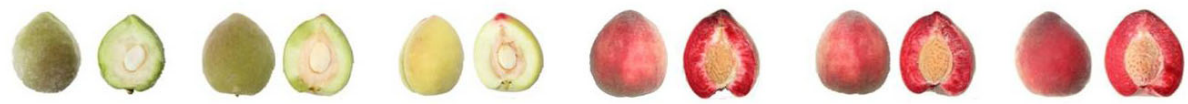

(c)
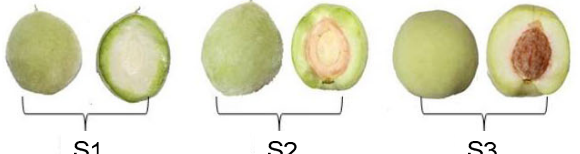

S3

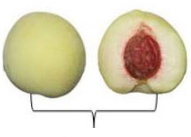

S4

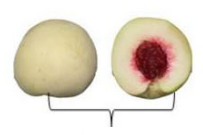

S5

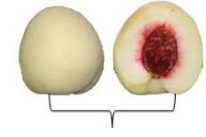

S6

Fig. 1 Changes of color in the mesocarp of three peach cultivars during fruit development

(a) Beijingyixianhong; (b) Heiyoutao; (c) Xuebaitao. Samples were assayed at 50 (S1), 64 (S2), 72 (S3), 80 (S4), 82 (S5), and 87 (S6) d after full bloom (DAFB) from 'Beijingyixianhong', and at 60 (S1), 74 (S2), 88 (S3), 97 (S4), 109 (S5), and 123 (S6) DAFB from both 'Heiyoutao' and 'Xuebaitao'

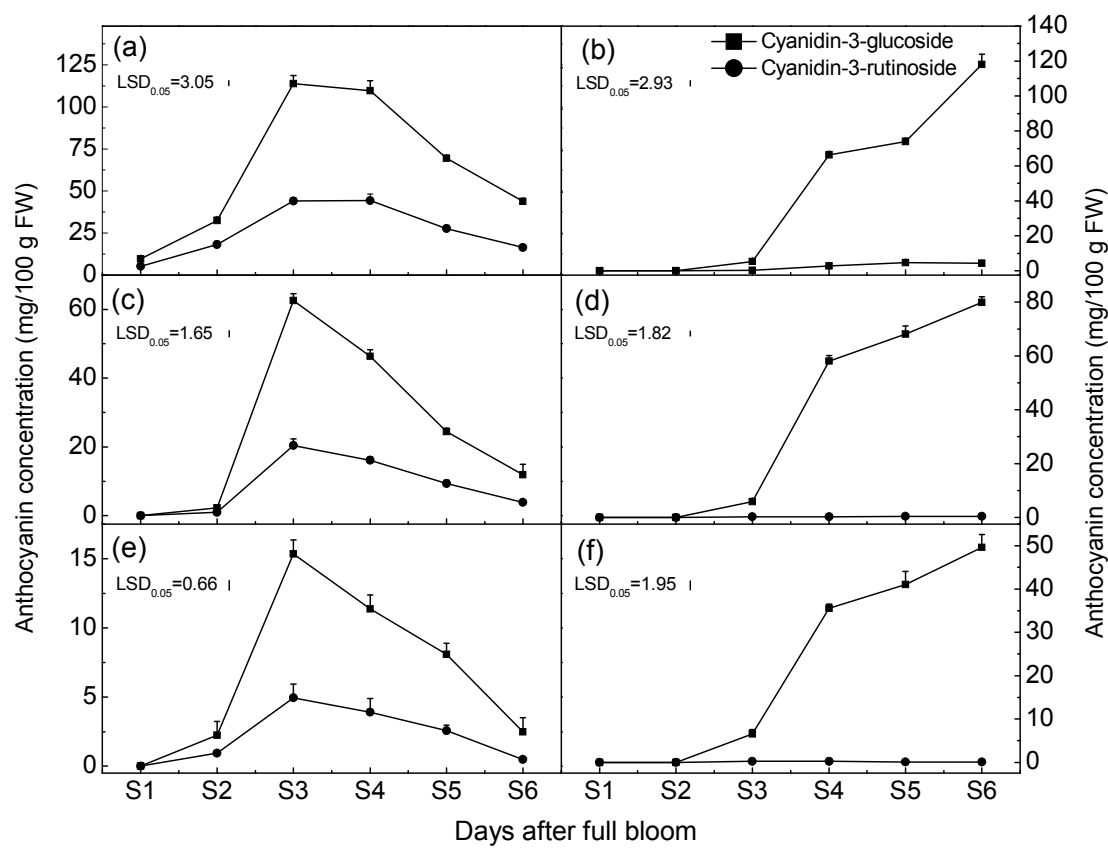

Fig. 2 Anthocyanin concentration in the fruit of 'Heiyoutao' (a, c, e) and 'Beijingyixianhong' (b, d, f) during fruit development, as measured by HPLC

$(a, b)$ Fruit skin; (c, d) Outer layer of mesocarp; (e, f) Inner layer of mesocarp. Fruit skin and flesh samples were collected separately from at least 12 replicate fruit of each cultivar. The vertical bars represent the standard error (SE) of three replicates

the mesocarp. We found that cyanidin-3-glucoside was the major anthocyanin in all layers of the fruit. Cyanidin-3-rutinoside was not detected in the mesocarp of 'Beijingyixianhong' and only small amounts were found in the fruit skin. Interestingly, the leaf midrib of 'Heiyoutao' contained both cyanidin-3glucoside and cyanidin-3-rutinoside, as did its fruit (Fig. S1). 'Xuebaitao', which produces fruits with a red color surrounding the stone during the mature stage, expressed only cyanidin-3-glucoside (Fig. S2).

\subsection{Expression of anthocyanin biosynthetic genes in two blood-flesh peach cultivars}

Nine genes were differentially expressed in the peach cultivars (Fig. 3). Firstly, the transcription of $P p P A L, P p U F G T$, and $P p M Y B 10$ reached peak levels during the initial stages of fruit development at $\mathrm{S} 2$ or S3. During this time, the expression levels of PpUFGT and PpMYB10 were higher in 'Beijingyixianhong' than in 'Heiyoutao'. Post-peak levels, expression of these genes continuously declined until maturity. 


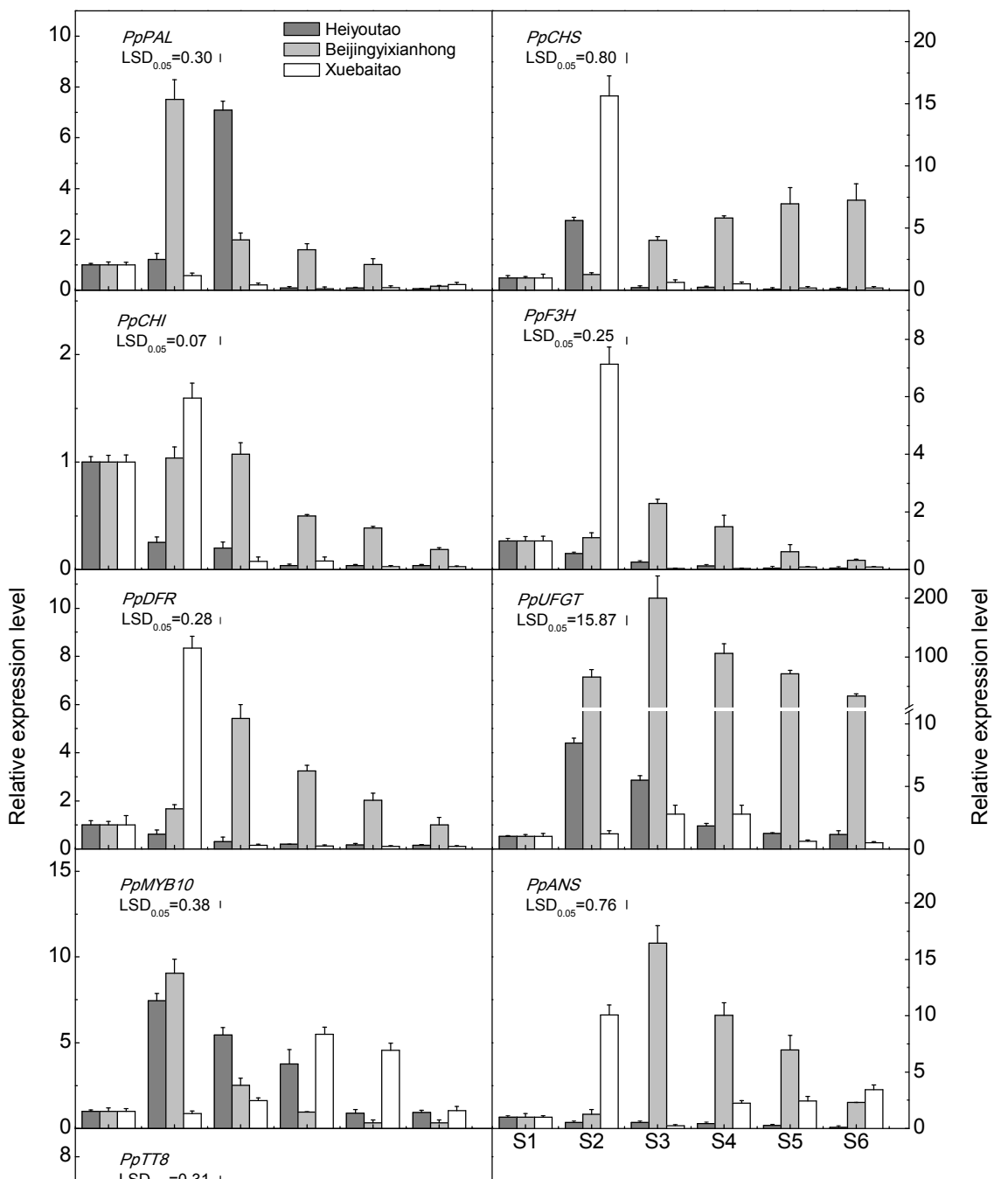

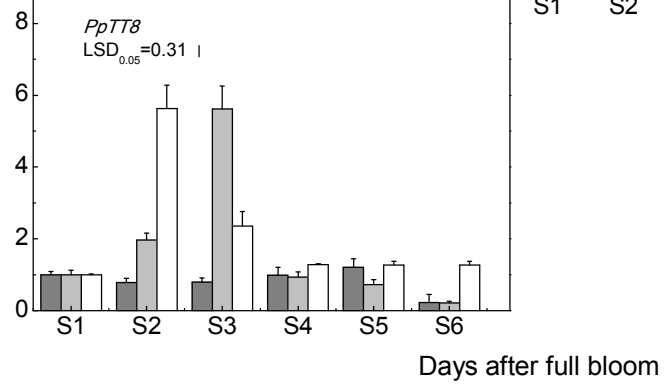

Secondly, the transcription of $P p C H I, P p F 3 H$, PpDFR, PpANS, and PpTT8 reached peak levels during the initial stages of fruit development at $\mathrm{S} 2$ or $\mathrm{S} 3$ in 'Beijingyixianhong' and 'Xuebaitao'. However, the overall expression of these genes in 'Heiyoutao' was very low and gradually declined throughout development. Finally, the expression of the gene PpCHS was closely related to the anthocyanin content in 'Beijingyixianhong', but had a tendency to continuously decline to maturity in 'Heiyoutao'. The expression of the gene PpCHS reached its peak level
Fig. 3 Expression analysis of anthocyanin biosynthetic genes in the mesocarp of 'Heiyoutao', 'Beijingyixianhong', and 'Xuebaitao' during fruit development

Expression levels of the $U B Q 10$ and TEF2 genes were used to normalize the expression of all other genes under identical conditions. The vertical bars represent the standard error (SE) of three replicates at S2 in the white-fleshed cultivar 'Xuebaitao'. This expression pattern was similar to that of 'Heiyoutao'.

\subsection{Expression of anthocyanin biosynthetic genes after the bag removal}

Following bag removal, anthocyanin accumulated in the mesocarp of the blood-flesh peach (Fig. 4) in a similar fashion to the natural state (as described in Fig. 2). Small amounts of anthocyanins were detected in the mesocarp of the 'Xuebaitao' fruit at 15 DABR. 
The transcription levels of seven genes $(P p P A L$, $P p C H I, P p F 3 H, P p D F R, P p M Y B 10, P p A N S$, and PpTT8) were markedly up-regulated in both bloodflesh peaches at 3 or 5 DABR, followed by a sharp drop (Fig. 5). The transcription levels of $P p P A L$ and PpTT8 were lower in the white-flesh of 'Xuebaitao', suggesting that these genes were non-sensitive to the bag removal treatment. Moreover, the transcription levels of $P p C H S$ and $P p U F G T$ still had relatively high expression in 'Beijingyixianhong' from 3 to 15 DABR, suggesting a common transcription mechanism that uses multiple pathways all contributing to the biosynthesis of anthocyanin.

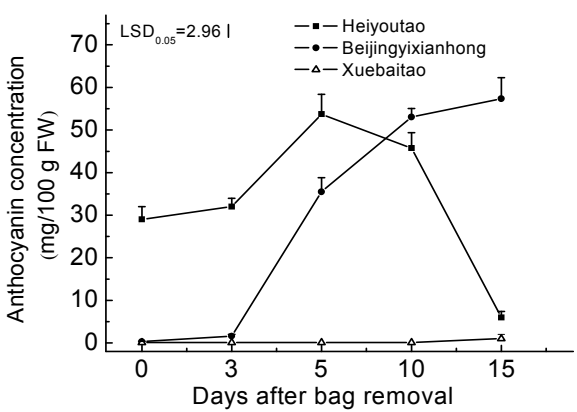

Fig. 4 Anthocyanin concentrations following bag removal in the mesocarp

Samples were assayed at $0,3,5,10$, and $15 \mathrm{~d}$ after bag removal (DABR). The vertical bars represent the standard error (SE) of three replicates

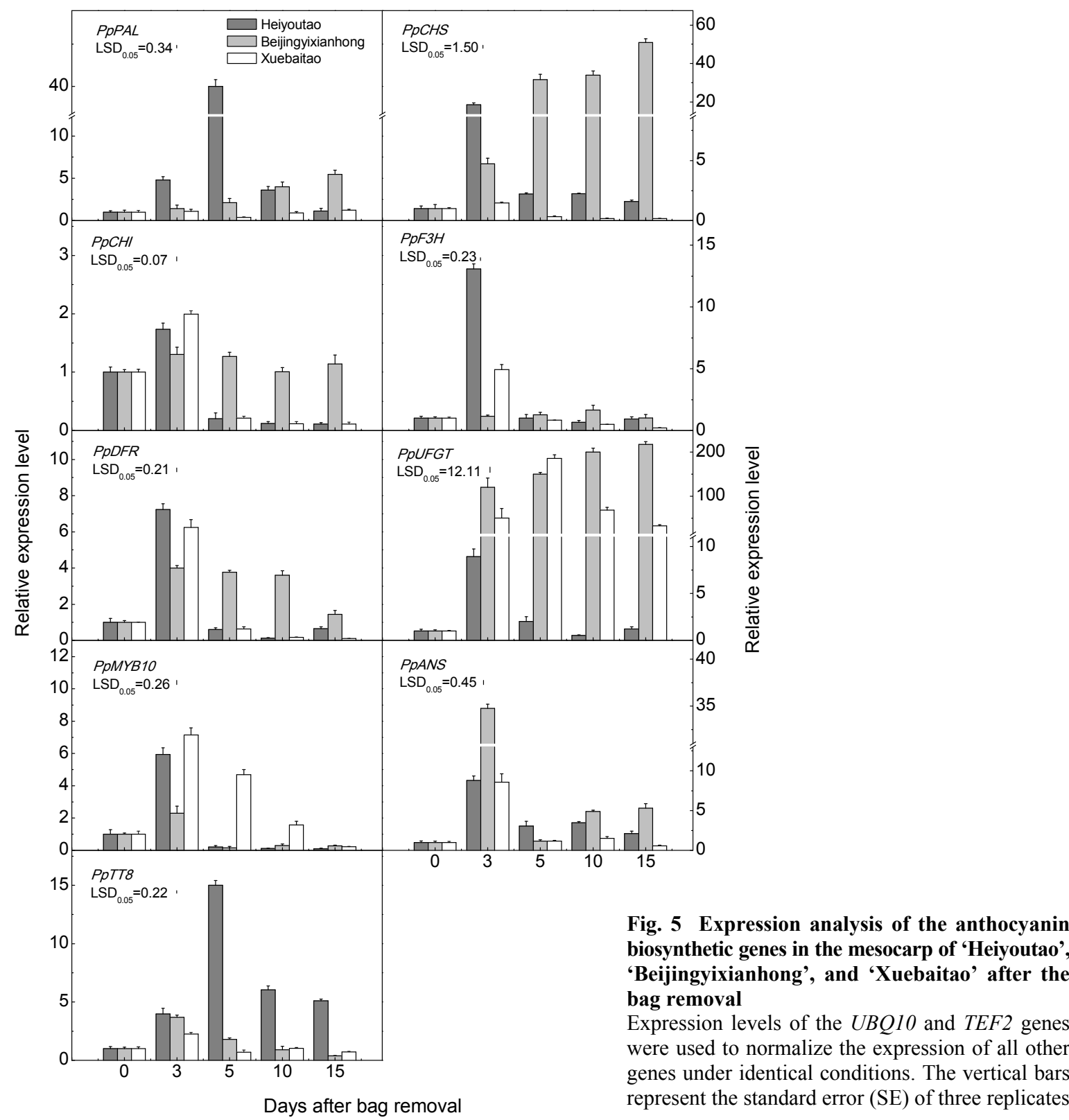




\subsection{Tissue-specific expression analysis}

Anthocyanin content varied greatly among the different organs and tissues of 'Heiyoutao', with the highest levels found in the petals and the lowest in the young stems (Fig. 6). The anthocyanin content in the petals was significantly higher in 'Heiyoutao' than in both 'Beijingyixianhong' and 'Xuebaitao'. These results are in accordance with visual observations (Fig. S3). Tissue-specific expression analysis revealed that the genes were differentially expressed in various organs and tissues (Fig. 7). Firstly, $P p C H S$, $P p U F G T$, and PpTT8 were all highly expressed in the fruit skin of 'Heiyoutao' and 'Beijingyixianhong'.

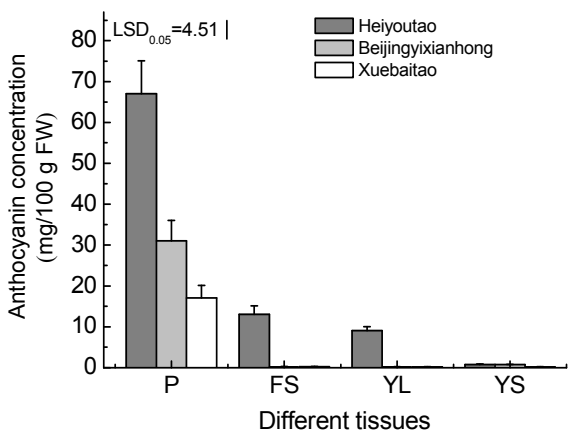

Fig. 6 Anthocyanin concentrations in various organs and tissues of the peach

P: petal; FS: fruit skin; YL: young leaf; YS: young stem. The vertical bars represent the standard error (SE) of three replicates

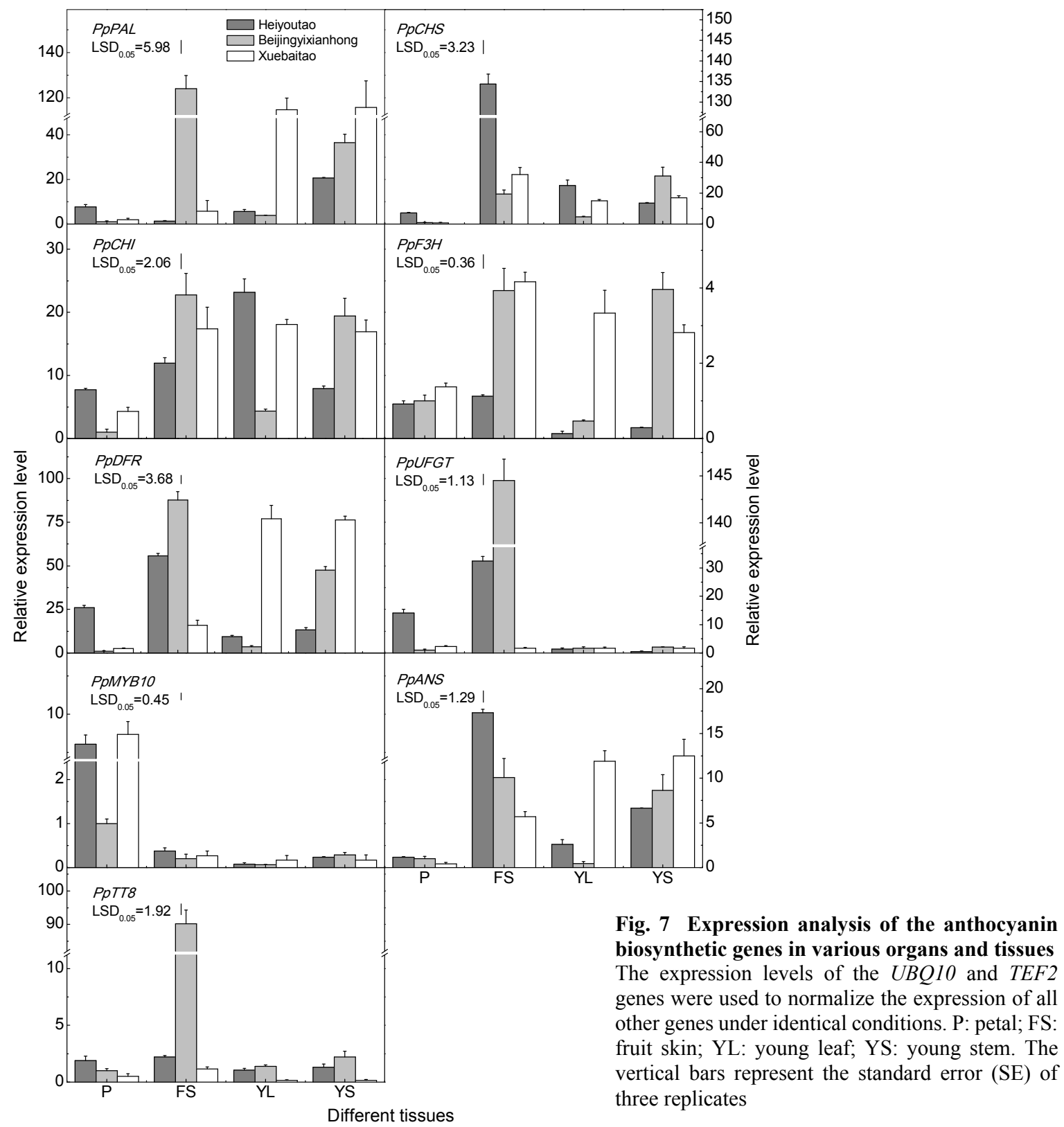


The transcription levels of the PpUFGT and PpTT8 genes were lower in 'Xuebaitao'. Secondly, $P p P A L$, $P p C H I, P p F 3 H, P p D F R$, and PpANS were all expressed at the highest level in the fruit skin and the young stems of 'Heiyoutao' and 'Beijingyixianhong'. These genes were also expressed at the highest level in the leaves of 'Xuebaitao', suggesting that the expression of the flavonoid "early" biosynthetic genes (EBGs) (including $P p P A L, P p F 3 H$, and $P p D F R$ ) is less associated with anthocyanin levels. Finally, PpMYB10 was expressed at the highest level in the petals and was highly correlated with anthocyanin levels in different tissues.

\section{Discussion}

Anthocyanin concentrations have previously been measured by ultraviolet spectrometry in the peach (Leng et al., 2000; Zhou et al., 2013). However, these studies showed the difficulty in distinguishing different anthocyanin components, as their concentrations across different organs and/or tissues were not identifiable. In the current study, we identified that cyaniding-3-glucoside is the major anthocyanin in the leaves, petals, and stems of the peach, consistent with previous studies (Cheng and Crisosto, 1997; Cevallos-Casals et al., 2006; Orazem et al., 2011). On the other hand, cyaniding-3-rutinoside was found in the fruit skin of 'Heiyoutao' and was nondetectable in the mesocarp of 'Beijingyixianhong', suggesting that production of this anthocyanin component differs among cultivars. Furthermore, we estimate that there are six types of anthocyanin pigments in the petals of these peach cultivars, making them much more complex than other organs or tissues (data not shown). However, the molecular mechanisms of anthocyanin biosynthesis are still not fully understood.

In this study, the expression of $P p C H S$ was found to correlate with the accumulation of anthocyanin, suggesting that it is a key gene involved in the process of anthocyanin biosynthesis. These findings also echo previous research (Tsuda et al., 2004), and thus this pattern may be common to other red fruit of the Rosaceae family. We found that the transcription levels of $P p P A L$ and $P p U F G T$ were much lower in the white-flesh cultivar 'Xuebaitao' than in the blood-flesh peaches. Taken together, this pattern of mRNA expression suggests that $P p U F G T$ is under a different regulatory regime, compared with the other flavonoid pathway genes in the peach, and that anthocyanin synthesis is controlled at a later stage than seen in previously studies (Boss et al., 1996; Kobayashi et al., 2001). In addition, these results suggest that anthocyanin biosynthesis is not active in the mesocarp of the white-flesh peach due to the absence of $P p P A L$. In contrast, the early-expressing genes of the flavonoid pathway (PpCHS, $P p F 3 H$, and $P p D F R)$ were all expressed in the white-flesh peach 'Xuebaitao' and are therefore likely to be involved in the synthesis of other secondary metabolites, such as free-auxins (Schwalm et al., 2003; Shen et al., 2013). Similar results have been found in grape (Boss et al., 1996), apple (Kim et al., 2003), Chinese bayberry (Niu et al., 2010), kiwifruit (Montefiori et al., 2011), and litchi (Wei et al., 2011). Furthermore, Schwalm et al. (2003) reported that $C H S$-dependent flavonoid aglycones have the potential to be endogenous regulators of the basipetal auxin flux, thereby leading to free-auxin accumulation. 'Heiyoutao' has the genotype $b f$, and the blood-flesh phenotype has been associated with reduced tree height in progeny derived from this cultivar (Shen et al., 2013). This suggests that the phenotype may be closely related to auxin flux, but the detailed mechanisms remain to be elucidated.

Results show that bagging has a significant effect on anthocyanin synthesis (Dussi et al., 1995). As expected, anthocyanin concentrations were generally higher in the natural state than following bagging. After bag removal, the transcription levels of most of the anthocyanin biosynthetic genes were markedly enhanced at 3 DABR, except for PpPAL and PpTT8. These results indicate that a single gene is not responsible for anthocyanin accumulation and that the coordinated action of many genes is likely involved in anthocyanin biosynthesis. This hypothesis is supported by previous findings in apple (Honda et al., 2002; Takos et al., 2006), pear (Yu et al., 2012), and grape (Walker et al., 2007). In particular, PpUFGT was clearly expressed in the white-flesh peach 'Xuebaitao', but anthocyanin accumulation was not detected (Figs. 4 and 5). These findings suggest that 
$P p U F G T$ is likely more highly regulated than the other flavonoid genes. Furthermore, Ravaglia et al. (2013) reported that $P p M Y B 10$ positively regulates the promoters of $P p U F G T$ and $P p D F R$. Our qPCR results show that the expression pattern of the $P p M Y B 10$ gene is highly correlated with $P p U F G T$ and PpDFR in 'Heiyoutao', but not in 'Beijingyixianhong'. These results suggest that $P$ PMYB10 plays a different role in the anthocyanin regulation of the two blood-flesh peaches. Moreover, some members of MYB10 are thought to be involved in regulating anthocyanin biosynthesis (Rahim et al., 2014; Uematsu et al., 2014). Further studies should focus on their responsiveness to both developmental and environmental stimuli in blood-flesh peaches.

Finally, in the two blood-flesh peach cultivars that we investigated, the petals were pink, owing to a greater accumulation of anthocyanins compared with other tissues (Fig. S3). Tissue-specific expression analysis revealed that $P p M Y B 10$ expression seemed more pronounced in petals than in other tissues (Fig. 7), strongly indicating that $P p M Y B 10$ is a key regulatory gene involved in anthocyanin biosynthesis within the petals of these peach cultivars. In contrast, the other anthocyanin biosynthesis genes ( $P p P A L, P p C H I$, $p P p F 3 H, P p D F R$, and $P p A N S$ ) were all highly expressed in the stems and leaves of the white-flesh peach 'Xuebaitao', even though anthocyanin was not detected. This result indicates that these genes are likely involved in processes other than anthocyanin production.

In conclusion, this study demonstrated large differences in anthocyanin accumulation among the two blood-flesh peaches studied. The transcription level of $P p C H S$ was closely related to anthocyanin accumulation, due to its diverse roles in the biosynthetic pathway. Further studies are required to elucidate the molecular mechanisms of action of PpCHS in the anthocyanin biosynthesis pathway.

\section{Acknowledgements}

We would like to thank Dr. Rang-jin XIE (Southwest University, China) for his comments on the manuscript.

\section{Compliance with ethics guidelines}

Yun JIAO, Rui-juan MA, Zhi-jun SHEN, Juan YAN, and Ming-liang YU declare that they have no conflict of interest.

This article does not contain any studies with human or animal subjects performed by any of the authors.

\section{References}

Allan, A.C., Hellens, R.P., Laing, W.A., 2008. MYB transcription factors that colour our fruit. Trends Plant Sci., 13(3):99-102. [doi:10.1016/j.tplants.2007.11.012]

Baudry, A., Heim, M.A., Dubreucq, B., et al., 2004. TT2, TT8, and TTG1 synergistically specify the expression of $B A N Y U L S$ and proanthocyanidin biosynthesis in Arabidopsis thaliana. Plant J., 39(3):366-380. [doi:10.1111/j. 1365-313X.2004.02138.x]

Baudry, A., Caboche, M., Lepiniec, L., 2006. TT8 controls its own expression in a feedback regulation involving TTG1 and homologous MYB and bHLH factors, allowing a strong and cell-specific accumulation of flavonoids in Arabidopsis thaliana. Plant J., 46(5):768-779. [doi:10. 1111/j.1365-313X.2006.02733.x]

Boss, P.K., Davies, C., Robinson, S.P., 1996. Expression of anthocyanin biosynthesis pathway genes in red and white grapes. Plant Mol. Biol., 32(3):565-569. [doi:10.1007/ BF00019111]

Butelli, E., Titta, L., Giorgio, M., et al., 2008. Enrichment of tomato fruit with health-promoting anthocyanins by expression of select transcription factors. Nat. Biotechnol., 26(11):1301-1308. [doi:10.1038/nbt.1506]

Cevallos-Casals, B.A., Byrne, D., Okie, W.R., et al., 2006. Selecting new peach and plum genotypes rich in phenolic compounds and enhanced functional properties. Food Chem., 96(2):273-280. [doi:10.1016/j.foodchem.2005.02. 032]

Chagné, D., Carlisle, C.M., Blond, C., et al., 2007. Mapping a candidate gene (MdMYB10) for red flesh and foliage colour in apple. BMC Genomics, 8(1):212 [doi:10.1186/ 1471-2164-8-212]

Chaparro, J., Werner, D., Whetten, R., et al., 1995. Inheritance, genetic interaction, and biochemical characterization of anthocyanin phenotypes in peach. J. Hered., 86(1):32-38.

Cheng, G.W., Crisosto, C.H., 1997. Iron-polyphenol complex formation and skin discoloration in peaches and nectarines. J. Amer. Soc. Hort. Sci., 122(1):95-99.

Dragsted, L.O., Krath, B., Ravn-Haren, G., et al., 2006. Biological effects of fruit and vegetables. Proc. Nutr. Soc., 65(1):61-67. [doi:10.1079/PNS2005480]

Dussi, M.C., Sugar, D., Wrolstad, R.E., 1995. Characterizing and quantifying anthocyanins in red pears and the effect of light quality on fruit color. J. Amer. Soc. Hort. Sci., 120(5):785-789.

Espley, R.V., Hellens, R.P., Putterill, J., et al., 2007. Red colouration in apple fruit is due to the activity of the MYB transcription factor, MdMYB10. Plant J., 49(3):414-427. [doi:10.1111/j.1365-313X.2006.02964.x]

Honda, C., Kotoda, N., Wada, M., et al., 2002. Anthocyanin biosynthetic genes are coordinately expressed during red coloration in apple skin. Plant Physiol. Biochem., 40(11): 955-962. [doi:10.1016/S0981-9428(02)01454-7]

Hsia, C.L., Luh, B.S., Chichester, C.O., 1965. Anthocyanin in freestone peaches. J. Food Sci., 30(1):5-12. [doi:10.1111/ j.1365-2621.1965.tb00253.x] 
Kim, S.H., Lee, J.R., Hong, S.T., et al., 2003. Molecular cloning and analysis of anthocyanin biosynthesis genes preferentially expressed in apple skin. Plant Sci., 165(2):403-413. [doi:10.1016/S0168-9452(03)00201-2]

Kobayashi, S., Ishimaru, M., Ding, C., et al., 2001. Comparison of UDP-glucose: flavonoid 3-O-glucosyltransferase (UFGT) gene sequences between white grapes (Vitis vinifera) and their sports with red skin. Plant Sci., 160(3):543-550. [doi:10.1016/S0168-9452(00)00425-8]

Leng, P., Itamura, H., Yamamura, H., et al., 2000. Anthocyanin accumulation in apple and peach shoots during cold acclimation. Sci. Hort., 83(1):43-50. [doi:10.1016/S03044238(99)00065-5]

Lepiniec, L., Debeaujon, I., Routaboul, J.M., et al., 2006. Genetics and biochemistry of seed flavonoids. Annu. Rev. Plant Biol., 57(1):405-430. [doi:10.1146/annurev.arplant. 57.032905.105252]

Lin-Wang, K., Bolitho, K., Grafton, K., et al., 2010. An R2R3 MYB transcription factor associated with regulation of the anthocyanin biosynthetic pathway in Rosaceae. $B M C$ Plant Biol., 10(1):50. [doi:10.1186/1471-2229-10-50]

Livak, K.J., Schmittgen, T.D., 2001. Analysis of relative gene expression data using real-time quantitative PCR and the $2^{-\Delta \Delta C_{\mathrm{T}}}$ method. Methods, 25(4):402-408. [doi:10.1006/ meth.2001.1262]

Montefiori, M., Espley, R.V., Stevenson, D., et al., 2011. Identification and characterisation of F3GT1 and F3GGT1, two glycosyltransferases responsible for anthocyanin biosynthesis in red-fleshed kiwifruit (Actinidia chinensis). Plant J., 65(1):106-118. [doi:10.1111/j.1365-313X.2010. 04409.x]

Morita, Y., Saitoh, M., Hoshino, A., et al., 2006. Isolation of cDNAs for R2R3-MYB, bHLH and WDR transcriptional regulators and identification of $c$ and $c a$ mutations conferring white flowers in the Japanese morning glory. Plant Cell Physiol., 47(4):457-470. [doi:10.1093/pcp/ pcj012]

Niu, S.S., Xu, C.J., Zhang, W.S., et al., 2010. Coordinated regulation of anthocyanin biosynthesis in Chinese bayberry (Myrica rubra) fruit by a R2R3 MYB transcription factor. Planta, 231(4):887-899. [doi:10.1007/s00425009-1095-z]

Orazem, P., Stampar, F., Hudina, M., 2011. Quality analysis of 'Redhaven' peach fruit grafted on 11 rootstocks of different genetic origin in a replant soil. Food Chem., 124(4): 1691-1698. [doi:10.1016/j.foodchem.2010.07.078]

Pandey, A., Misra, P., Khan, M.P., et al., 2014. Co-expression of Arabidopsis transcription factor, AtMYB12, and soybean isoflavone synthase, GmIFS1, genes in tobacco leads to enhanced biosynthesis of isoflavones and flavonols resulting in osteoprotective activity. Plant Biotechnol. J., 12(1):69-80. [doi:10.1111/pbi.12118]

Rahim, M.A., Busatto, N., Trainotti, L., 2014. Regulation of anthocyanin biosynthesis in peach fruits. Planta, 1-17. [doi:10.1007/s00425-014-2078-2]

Ravaglia, D., Espley, R.V., Henry-Kirk, R.A., et al., 2013.
Transcriptional regulation of flavonoid biosynthesis in nectarine (Prunus persica) by a set of R2R3 MYB transcription factors. BMC Plant Biol., 13(1):68. [doi:10. 1186/1471-2229-13-68]

Schwalm, K., Aloni, R., Langhans, M., et al., 2003. Flavonoidrelated regulation of auxin accumulation in Agrobacterium tumefaciens-induced plant tumors. Planta, 218(2):163-178. [doi:10.1007/s00425-003-1104-6]

Shen, Z., Confolent, C., Lambert, P., et al., 2013. Characterization and genetic mapping of a new blood-flesh trait controlled by the single dominant locus $D B F$ in peach. Tree Genet. Genomes, 9(6):1435-1446. [doi:10.1007/ s11295-013-0649-1]

Shin, W.H., Park, S.J., Kim, E.J., 2006. Protective effect of anthocyanins in middle cerebral artery occlusion and reperfusion model of cerebral ischemia in rats. Life Sci., 79(2):130-137. [doi:10.1016/j.lfs.2005.12.033]

Takos, A.M., Jaffe, F.W., Jacob, S.R., et al., 2006. Light-induced expression of a $M Y B$ gene regulates anthocyanin biosynthesis in red apples. Plant Physiol., 142(3):1216-1232. [doi:10.1104/pp.106.088104]

Tong, Z., Gao, Z., Wang, F., et al., 2009. Selection of reliable reference genes for gene expression studies in peach using real-time PCR. BMC Mol. Biol., 10(1):71. [doi:10. 1186/1471-2199-10-71]

Tsuda, T., Yamaguchi, M., Honda, C., et al., 2004. Expression of anthocyanin biosynthesis genes in the skin of peach and nectarine fruit. J. Amer. Soc. Hort. Sci., 129(6): 857-862.

Uematsu, C., Katayama, H., Makino, I., et al., 2014. Peace, a MYB-like transcription factor, regulates petal pigmentation in flowering peach 'Genpei' bearing variegated and fully pigmented flowers. J. Exp. Bot., 65(4):1081-1094. [doi:10.1093/jxb/ert456]

van Blaricom, L., Senn, T., 1967. Anthocyanin pigments in freestone peaches grown in the Southeast. Proc. Amer. Soc. Hort. Sci., 90:541-545.

Walker, A.R., Lee, E., Bogs, J., et al., 2007. White grapes arose through the mutation of two similar and adjacent regulatory genes. Plant J., 49(5):772-785. [doi:10.1111/j. 1365-313X.2006.02997.x]

Wei, Y.Z., Hu, F.C., Hu, G.B., et al., 2011. Differential expression of anthocyanin biosynthetic genes in relation to anthocyanin accumulation in the pericarp of Litchi chinensis Sonn. PLoS ONE, 6(4):e19455. [doi:10.1371/ journal.pone.0019455]

Williams, C.M., El Mohsen, M.A., Vauzour, D., et al., 2008. Blueberry-induced changes in spatial working memory correlate with changes in hippocampal CREB phosphorylation and brain-derived neurotrophic factor (BDNF) levels. Free Radic. Biol. Med., 45(3):295-305. [doi:10. 1016/j.freeradbiomed.2008.04.008]

Yu, B., Zhang, D., Huang, C., et al., 2012. Isolation of anthocyanin biosynthetic genes in red chinese sand pear (Pyrus pyrifolia Nakai) and their expression as affected by organ/tissue, cultivar, bagging and fruit side. Sci. Hort., 
136:29-37. [doi:10.1016/j.scienta.2011.12.026]

Zhang, X.D., Allan, A.C., Yi, Q.O., et al., 2011. Differential gene expression analysis of Yunnan red pear, Pyrus pyrifolia, during fruit skin coloration. Plant Mol. Biol. Rep., 29(2):305-314. [doi:10.1007/s11105-010-0231-z]

Zhou, Y., Guo, D., Li, J., et al., 2013. Coordinated regulation of anthocyanin biosynthesis through photorespiration and temperature in peach (Prunus persica f. atropurpurea). Tree Genet. Genomes, 9(1):265-278. [doi:10.1007/s11295012-0552-1]

\section{List of electronic supplementary materials}

Fig. S1 HPLC chromatogram of the anthocyanin components in the leaf midrib of 'Heiyoutao'

Fig. S2 HPLC chromatogram of the cultivar 'Xuebaitao' from the flesh surrounding the stone at maturity

Fig. S3 Petals during full bloom of 'Beijingyixianhong' (a), 'Heiyoutao' (b), and 'Xuebaitao' (c)

\section{中文概要:}

本文题目：两个红肉桃品种果实发育期花色素苷合成与分子调控研究

Gene regulation of anthocyanin biosynthesis in two blood-flesh peach (Prunus persica $(\mathrm{L}$. Batsch) cultivars during fruit development

研究目的: 深入分析两个红肉桃品种果实发育期花色素苷合成差异的分子机理, 为红肉桃品种的遗传改 良与利用提供理论依据。

创新要点: 阐明两个红肉桃品种果实发育期花色素苷生物的合成与相关基因的时空表达规律，探讨造成 两个品种花色素苷积累差异的内在分子机制。

研究方法: 应用高效液相色谱 (HPLC) 和逆转录聚合酶链式反应 (RT-PCR) 分别鉴定两个红肉桃品种 花色素苷的积累规律与合成相关基因的表达水平, 进一步探讨两者花色素苷合成的内在分子 调控机理。

重要结论: 两个红肉桃品种果实发育期花色素苷合成与 $P p C H S$ 表达水平密切相关, 同时 $P p P A L$ 在白肉 桃品种果实发育期的表达水平低于红肉桃品种, 推测这两种基因可能是限制白肉桃品种果实 中花色素苷合成的因素之一。

关键词组: 红肉桃; 花色素苷; 套袋; 基因表达 